PROCEEDINGS OF THE

AMERICAN MATHEMATICAL SOCIETY

Volume 128, Number 9, Pages 2705-2713

S 0002-9939(00)05375-2

Article electronically published on February 28, 2000

\title{
THE SCHUR ALGORITHM AND COEFFICIENT CHARACTERIZATIONS FOR GENERALIZED SCHUR FUNCTIONS
}

\author{
TIBERIU CONSTANTINESCU AND AURELIAN GHEONDEA
}

(Communicated by David R. Larson)

\begin{abstract}
In this paper we analyze the existence of a Schur algorithm and obtain coefficient characterizations for the functions in a generalized Schur class. An application to an interpolation problem of Carathéodory type raised by M.G. Krel̆n and H. Langer is indicated.
\end{abstract}

\section{INTRODUCTION}

Generalized Schur classes of functions have been introduced by M.G. Kreĭn and H. Langer in [9] and were intensively studied by them and many others. These classes appear as a natural extension of the classical setting of bounded analytic functions on the unit disk, and their investigation leads to new phenomena in interpolation and coefficient problems. A comprehensive study of Schur classes on Pontryagin spaces was recently undertaken by D. Alpay, A. Dijksma, J. Rovnyak, H. de Snoo in 2].

In this paper we deal with two questions formulated as open problems in [2]. Thus, Section 2 contains the solution to a question in [5] (see also [2], p. 186) concerning the Schur algorithm for generalized Schur classes. In Section 3 we provide a partial answer to another question in [5] (see also [2, p. 186) concerning the Carathéodory coefficient estimation. As an application, and based on our previous results in [6], we characterize the solvability of a generalized Carathéodory interpolation problem for matrix valued meromorphic functions, a problem raised in the scalar case by M.G. Kreĭn and H. Langer in [10].

\section{Generalized Schur Classes and the Schur algorithm}

A Krein space $\mathcal{U}$ is a complex linear space endowed with an inner product $[\cdot, \cdot]$ with the property that there exists a bounded linear operator $J$ on $\mathcal{U}$ such that $J^{*}=J^{-1}=J$ and the new inner product on $\mathcal{U}$ defined by $\langle h, g\rangle_{J}=[J h, g]$ for $h, g \in \mathcal{U}$, turns $\mathcal{U}$ into a Hilbert space. An operator $J$ as above is called a fundamental symmetry on $\mathcal{U}$. For each fundamental symmetry $J$ on $\mathcal{U}$, the decomposition

Received by the editors March 30, 1998 and, in revised form, October 29, 1998.

1991 Mathematics Subject Classification. Primary 30C50, 47B50, 30E05.

Key words and phrases. Schur functions, Schur algorithm, coefficient characterization, Carathéodory problem, Krel̆n space.

The second author's research was partially supported by the Ministry of Research and Technology of Romania grant 4022GR/1998. 
$\mathcal{U}=\mathcal{U}^{+} \oplus \mathcal{U}^{-}$holds, where $\mathcal{U}^{ \pm}=\operatorname{Range}(I \mp J)$, and it is called a fundamental decomposition. The cardinal numbers $\kappa^{ \pm}[\mathcal{U}]=\operatorname{dim}\left(\mathcal{U}^{ \pm}\right)$are independent on the chosen fundamental decomposition and are called positive/negative indices of $\mathcal{U}$. If $\kappa^{-}[\mathcal{U}]<\infty$, then $\mathcal{U}$ is called a Pontryagin space. For two Krein spaces $\mathcal{U}$ and $\mathcal{H}$ we denote by $\mathcal{U} \oplus \mathcal{H}$ the orthogonal direct sum Krel̆n space of $\mathcal{U}$ and $\mathcal{H}$; if $\mathcal{G}$ is a Krein subspace of $\mathcal{U}$, then $\mathcal{U} \ominus \mathcal{G}$ denotes the orthogonal complement of $\mathcal{G}$ in $\mathcal{U}$. We denote by $\mathcal{L}(\mathcal{U}, \mathcal{Y})$ the set of bounded linear operators from $\mathcal{U}$ into $\mathcal{Y}$. For an operator $T \in \mathcal{L}(\mathcal{U}, \mathcal{Y})$ we use the notation $T^{\sharp} \in \mathcal{L}(\mathcal{Y}, \mathcal{U})$ for the adjoint of $T$, that is, $[T h, g]=\left[h, T^{\sharp} g\right]$ for $h \in \mathcal{U}, g \in \mathcal{Y}$. The operator $U$ is unitary if $U^{\sharp}=U^{-1}$.

Given a selfadjoint operator $A \in \mathcal{L}(\mathcal{K}), \mathcal{K}$ a Kreĭn space, the negative index $\kappa^{-}(A)$ is defined as the dimension of the spectral subspace corresponding to the interval $(-\infty, 0)$ of the selfadjoint operator $J A$ in the Hilbert space $\left(\mathcal{K},\langle\cdot, \cdot\rangle_{J}\right)$, where $J$ is an arbitrary fundamental symmetry on $\mathcal{K}$. If $\kappa^{-}(A)<\infty$, then the negative index is equal to the number of negative eigenvalues, counted according to their multiplicities, of the operator $J A$; in this case, $\kappa^{-}(A)$ also equals the number of the negative squares of the quadratic form $\mathcal{K} \ni x \mapsto[A x, x]$.

We now recall the definition of the generalized classes of Schur functions. For a function $S$ defined on a domain in the unit disk containing the origin and whose values are operators in $\mathcal{L}(\mathcal{U}, \mathcal{Y})$, let $\tilde{S}(z)=S^{\sharp}(\bar{z})$, and consider the kernels

$$
K_{S}(w, z)=\frac{I-S(z) S^{\sharp}(w)}{1-z \bar{w}}, \quad D_{S}(w, z)=\left[\begin{array}{cc}
K_{S}(w, z) & \frac{S(z)-S(\bar{w})}{z-\bar{w}} \\
\frac{\tilde{S}(z)-\tilde{S}(\bar{w})}{z-\bar{w}} & K_{\tilde{S}}(w, z)
\end{array}\right],
$$

defined for all $w$ and $z$ for which the above relations make sense. Given a nonnegative integer $\kappa$ and $\mathcal{U}, \mathcal{Y}$ Krel̆n spaces, the (generalized) Schur class $\mathcal{S}_{\kappa}(\mathcal{U}, \mathcal{Y})$ consists of all holomorphic functions in a neighbourhood of the origin, with the property that the four kernels $K_{S}, D_{S}, K_{\tilde{S}}, D_{\tilde{S}}$ all have $\kappa$ negative squares. As pointed out in [2], the class $\mathcal{S}_{\kappa}(\mathcal{U}, \mathcal{Y})$, for $\mathcal{U}$ and $\mathcal{Y}$ Pontryagin spaces of the same negative index, appears as an interesting class of functions for which much of the classical theory (i.e. $\mathcal{U}, \mathcal{Y}$ Hilbert spaces and $\kappa=0$ ) is expected to be recovered. However, it happens that a key structure theorem related to the so-called Schur algorithm does not hold for these generalized Schur classes.

The classical Schur algorithm starts with a function $f$ holomorphic and bounded by one on the unit open disk of the complex plane. A sequence of functions of the same type is generated by the formulas $f_{0}(z)=f(z)$ and

$$
f_{n+1}(z)=\frac{f_{n}(z)-f_{n}(0)}{z\left(1-\overline{f_{n}(z)} f_{n}(z)\right)}, \quad n \geq 0
$$

The key point is that the numbers $f_{n}(0), n \geq 0$, usually known as Schur parameters, uniquely determine the function $f$. This continued fractions-type algorithm has a number of applications, e.g. as mentioned in [5], 2], and their references.

An extension of this algorithm to the generalized Schur classes appears to be of interest. As explained in [5] and [2], the question is as follows: suppose that $S$ belongs to $\mathcal{S}_{\kappa}(\mathcal{U}, \mathcal{Y}), \kappa^{-}[\mathcal{U}]=\kappa^{-}[\mathcal{Y}]$, and $D=S(0)$ satisfies the relation $\kappa^{-}\left(I-D D^{\sharp}\right)=\kappa^{-}\left(I-D^{\sharp} D\right)=\kappa_{0} \leq \kappa$. Then choose a Julia operator associated to $D$, that is, a unitary operator

$$
U=\left[\begin{array}{ll}
D & \beta \\
\gamma & \delta
\end{array}\right]: \mathcal{U} \oplus \mathcal{D} \rightarrow \mathcal{Y} \oplus \tilde{\mathcal{D}}
$$


such that $\beta$ (equivalently, $\gamma^{\sharp}$ ) has zero kernel. It is asked in [5] (see also 2]) whether there exists a function $S_{1} \in \mathcal{S}_{\kappa-\kappa_{0}}(\tilde{\mathcal{D}}, \mathcal{D})$ such that

$$
S(z)=D+z \beta S_{1}(z)\left(I-z \delta S_{1}(z)\right)^{-1} \gamma
$$

in a neighbourhood of the origin. The connection of this question with the classical Schur algorithm is explained in detail in [5] and [2]. The answer to this question is negative in general, as shown in [7] by an example in the case $\kappa_{0}<\kappa$. We describe this example here for the sake of completeness. The motivation for this construction is given in [7]. Set $\mathcal{U}=\mathbb{C}^{3}$, with the Euclidean structure, and consider $\mathcal{H}$ the Pontryagin space of negative index 1 obtained on $\mathbb{C}^{3}$ with respect to the fundamental symmetry

$$
\left[\begin{array}{lll}
1 & 0 & 0 \\
0 & 0 & 1 \\
0 & 1 & 0
\end{array}\right]
$$

Then, define the operators $A, B, C, D$ as given by the matrices:

$$
A=\left[\begin{array}{ccc}
0 & 0 & 0 \\
0 & 0 & -1 \\
0 & -1 & 1
\end{array}\right], B=\left[\begin{array}{lll}
1 & 0 & 0 \\
0 & 0 & 0 \\
0 & 1 & 1
\end{array}\right], C=\left[\begin{array}{lll}
1 & 0 & 0 \\
0 & 0 & 1 \\
0 & 0 & 1
\end{array}\right], D=\left[\begin{array}{lll}
0 & 0 & 0 \\
0 & 1 & 0 \\
0 & 0 & 1
\end{array}\right]
$$

We define the function $S(z)=D+z C(I-z A)^{-1} B$ holomorphic in a neighbourhood of the origin. One checks that $S \in \mathcal{S}_{1}(\mathcal{U})$ and we notice that $\kappa^{-}\left(I-S(0) S^{*}(0)\right)=$ $\kappa^{-}\left(I-S^{*}(0) S(0)\right)=0$. The computation of the Julia operator associated to $S(0)=$ $D$ (and which is essentially unique in this case) is easy. Thus, if we assume that there exists $S_{1} \in \mathcal{S}_{1}(\tilde{\mathcal{D}}, \mathcal{D})$ such that (2.1) holds, then we must have

$$
D+z C(I-z A)^{-1} B=D+z\left[\begin{array}{l}
1 \\
0 \\
0
\end{array}\right] S_{1}(z)\left[\begin{array}{lll}
1 & 0 & 0
\end{array}\right]
$$

for $z$ in some neighbourhood of the origin. We therefore must have

$$
C B=\left[\begin{array}{ccc}
S_{1}(0) & 0 & 0 \\
0 & 0 & 0 \\
0 & 0 & 0
\end{array}\right]
$$

which is not possible. We are left with the analysis of the case $\kappa_{0}=\kappa$.

Theorem 2.1. Let $S \in \mathcal{S}_{\kappa}(\mathcal{U}, \mathcal{Y})$ be a Schur function, where $\mathcal{U}$ and $\mathcal{Y}$ are Kreün spaces such that $\kappa^{-}[\mathcal{U}]=\kappa^{-}[\mathcal{Y}]$. Assume that

$$
\kappa^{-}\left(I-D D^{\sharp}\right)=\kappa^{-}\left(I-D^{\sharp} D\right)=\kappa,
$$

where $D=S(0)$, and choose a Julia operator $\left[\begin{array}{cc}D & \beta \\ \gamma & \delta\end{array}\right]: \mathcal{U} \oplus \mathcal{D} \rightarrow \mathcal{Y} \oplus \tilde{\mathcal{D}}$. Then there exists a function $S_{1} \in \mathcal{S}_{0}(\tilde{\mathcal{D}}, \mathcal{D})$ such that $S(z)=D+z \beta S_{1}(z)\left(I-z \delta S_{1}(z)\right)^{-1} \gamma$ for all $z$ in a neighbourhood of the origin.

Proof. By Theorems 5.3.16 and 5.3.18 in [3], and Theorem 2.1.2 in [2], it follows that there exists a unitary operator

$$
W=\left[\begin{array}{ll}
D & C \\
B & A
\end{array}\right]: \mathcal{U} \oplus \mathcal{H} \rightarrow \mathcal{Y} \oplus \mathcal{H}
$$


such that $\kappa^{-}[\mathcal{H}]=\kappa$ and $S(z)=D+z C(I-z A)^{-1} B$ for $z$ in a neighbourhood of the origin. Since the operator $\left[\begin{array}{l}D \\ B\end{array}\right]$ is isometric and $\kappa^{-}\left(I-D D^{\sharp}\right)=\kappa^{-}[\mathcal{H}]=\kappa<\infty$, it follows from Lemma 2.2 in $[$, Part I, that $B=V \gamma$, where $V \in \mathcal{L}(\tilde{\mathcal{D}}, \mathcal{H})$ is isometric. Since $\kappa^{-}[\tilde{\mathcal{D}}]=\kappa^{-}[\mathcal{H}]=\kappa$, it follows that $V=\left[\begin{array}{c}\tilde{V} \\ 0\end{array}\right]: \tilde{D} \rightarrow \mathcal{H}_{1} \oplus \mathcal{R}_{1}$, where $\tilde{V} \in \mathcal{L}\left(\tilde{\mathcal{D}}, \mathcal{H}_{1}\right)$ is a unitary operator and $\mathcal{R}_{1}=\operatorname{ker} V^{\sharp}$ is a Hilbert space. Therefore, $W$ has the matrix representation

$$
\begin{gathered}
W=\left[\begin{array}{cc}
D & C \\
B & A
\end{array}\right]=\left[\begin{array}{ccc}
D & a & b \\
\tilde{V} \gamma & A_{11} & A_{12} \\
0 & A_{21} & A_{22}
\end{array}\right] \\
=\left[\begin{array}{ccc}
I & 0 & 0 \\
0 & \tilde{V} & 0 \\
0 & 0 & I
\end{array}\right]\left[\begin{array}{ccc}
D & a & b \\
\gamma & \tilde{V}^{\sharp} A_{11} \tilde{V} & \tilde{V}^{\sharp} A_{12} \\
0 & A_{21} \tilde{V} & A_{22}
\end{array}\right]\left[\begin{array}{ccc}
I & 0 & 0 \\
0 & \tilde{V}^{\sharp} & 0 \\
0 & 0 & I
\end{array}\right] .
\end{gathered}
$$

The operator with the matrix representation

$$
\left[\begin{array}{ccc}
D & a & b \\
\gamma & \tilde{V}^{\sharp} A_{11} \tilde{V} & \tilde{V}^{\sharp} A_{12} \\
0 & A_{21} \tilde{V} & A_{22}
\end{array}\right]
$$

is unitary from $\mathcal{U} \oplus \tilde{D} \oplus \mathcal{R}_{1}$ onto $\mathcal{Y} \oplus \tilde{D} \oplus \mathcal{R}_{1}$. Multiplying this operator to the left by the unitary operator

$$
\left[\begin{array}{ccc}
D^{\sharp} & \gamma^{\sharp} & 0 \\
\beta^{\sharp} & \delta^{\sharp} & 0 \\
0 & 0 & I
\end{array}\right]
$$

we obtain a unitary operator with matrix representation

$$
\left[\begin{array}{lll}
I & X & Y \\
0 & D^{\prime} & C^{\prime} \\
0 & B^{\prime} & A^{\prime}
\end{array}\right]
$$

It follows that necessarily $X=0$ and $Y=0$, and hence

$$
\left[\begin{array}{cc}
D & C \\
B & A
\end{array}\right]=\left[\begin{array}{ccc}
I & 0 & 0 \\
0 & \tilde{V} & 0 \\
0 & 0 & I
\end{array}\right]\left[\begin{array}{ccc}
D & \beta & 0 \\
\gamma & \delta & 0 \\
0 & 0 & I
\end{array}\right]\left[\begin{array}{ccc}
I & 0 & 0 \\
0 & D^{\prime} & C^{\prime} \\
0 & B^{\prime} & A^{\prime}
\end{array}\right]\left[\begin{array}{ccc}
I & 0 & 0 \\
0 & \tilde{V}^{\sharp} & 0 \\
0 & 0 & I
\end{array}\right]
$$

where

$$
W^{\prime}=\left[\begin{array}{ll}
D^{\prime} & C^{\prime} \\
B^{\prime} & A^{\prime}
\end{array}\right]: \tilde{\mathcal{D}} \oplus \mathcal{R}_{1} \rightarrow \mathcal{D} \oplus \mathcal{R}_{1}
$$

is a unitary operator. The function $S_{1}(z)=D^{\prime}+z C^{\prime}\left(I-z A^{\prime}\right)^{-1} B^{\prime}$ is holomorphic in a neighbourhood of the origin. Moreover, we obtain from (2.3) that

$$
C=\left[\begin{array}{ll}
\beta D^{\prime} \tilde{V}^{\sharp} & \beta C^{\prime}
\end{array}\right], \quad B=\left[\begin{array}{c}
\tilde{V} \gamma \\
0
\end{array}\right], \quad A=\left[\begin{array}{cc}
\tilde{V} \delta D^{\prime} \tilde{V}^{\sharp} & \tilde{V} \delta C^{\prime} \\
B^{\prime} \tilde{V}^{\sharp} & A^{\prime}
\end{array}\right] .
$$


Using a well-known formula for the inversion of a $2 \times 2$ matrix, we deduce that

$$
\begin{aligned}
S(z)= & D+z \beta\left[\begin{array}{cc}
D^{\prime} & C^{\prime}
\end{array}\right]\left[\begin{array}{cc}
\tilde{V}^{\sharp} & 0 \\
0 & I
\end{array}\right] \\
& \times\left(I-z\left[\begin{array}{cc}
\tilde{V} & 0 \\
0 & I
\end{array}\right]\left[\begin{array}{cc}
\delta D^{\prime} & \delta C^{\prime} \\
B^{\prime} & A^{\prime}
\end{array}\right]\left[\begin{array}{cc}
\tilde{V^{\sharp}} & 0 \\
0 & I
\end{array}\right]\right)^{-1}\left[\begin{array}{cc}
\tilde{V} & 0 \\
0 & I
\end{array}\right]\left[\begin{array}{l}
\gamma \\
0
\end{array}\right] \\
= & D+z \beta\left[\begin{array}{ll}
D^{\prime} & C^{\prime}
\end{array}\right]\left[\begin{array}{cc}
I-z \delta D^{\prime} & -z \delta C^{\prime} \\
-z B^{\prime} & I-z A^{\prime}
\end{array}\right]^{-1}\left[\begin{array}{l}
\gamma \\
0
\end{array}\right] \\
= & D+z \beta\left(D^{\prime} \Delta^{-1}+z C^{\prime}\left(I-z A^{\prime}\right)^{-1} B^{\prime} \Delta^{-1}\right) \gamma=D+z \beta S_{1}(z) \Delta^{-1} \gamma,
\end{aligned}
$$

where $\Delta=I-z \delta D^{\prime}-z^{2} \delta C^{\prime}\left(I-z A^{\prime}\right)^{-1} B^{\prime}=I-z \delta S_{1}(z)$. Therefore (2.1) holds, and taking into account Theorem 4.5.1 in [2] we deduce that $S_{1} \in \mathcal{S}_{0}(\tilde{\mathcal{D}}, \mathcal{D})$.

As a conclusion, we obtained the simple condition (2.2) that makes the Schur algorithm work in the generalized Schur classes. We also notice that if $\kappa=0$, then our Theorem 2.1 reduces to Theorem 2.2 in [5].

\section{Coefficient characterizations of the generalized Schur Classes}

In this section we are interested in obtaining a characterization of the functions in a Schur class in terms of their Taylor coefficients. Under the additional assumption of the convergence of the Taylor series in a neighbourhood of the origin, in the scalar case this problem was solved by M.G. Krel̆ and H. Langer in [10] and it was extended by T.Ya. Azizov; cf. [3], Theorem 3.16, p. 275. A general result of this type was obtained in [5] for the class $\mathcal{S}_{0}(\mathcal{U}, \mathcal{Y})$, with $\mathcal{U}$ and $\mathcal{Y}$ Kreln spaces. It appeared to be of interest to obtain similar results for the class $\mathcal{S}_{\kappa}(\mathcal{U}, \mathcal{Y})$, when $\mathcal{U}$ and $\mathcal{Y}$ are of dimension greater than 1 (see [5], [2], p. 186, for comments on this problem).

The proof of the result in [5] is based on the fact that a Schur-type algorithm exists for $\mathcal{S}_{0}(\mathcal{U}, \mathcal{Y})$, but as it was mentioned in the previous section, this is no longer the case for a Schur class with $\kappa>0$. However, we can show that a positive result can be obtained for the class $\mathcal{S}_{\kappa}(\mathcal{U}, \mathcal{Y})$ when $\kappa^{-}[\mathcal{U}]=\kappa^{-}[\mathcal{Y}]<\infty$. This case is sufficiently general to allow an application to a Carathéodory type problem of M.G. Krel̆n and H. Langer (see the next section).

Theorem 3.1. Let $\mathcal{U}$ and $\mathcal{Y}$ be Pontryagin spaces with $\kappa^{-}[\mathcal{U}]=\kappa^{-}[\mathcal{Y}]$, let

$$
S(z)=S_{0}+S_{1} z+S_{2} z^{2}+\ldots
$$

be a formal power series with coefficients in $\mathcal{L}(\mathcal{U}, \mathcal{Y})$ and consider the Toeplitz matrices

$$
T_{n}=\left[\begin{array}{cccc}
S_{0} & & & \\
S_{1} & S_{0} & 0 & \\
& \ddots & \ddots & \\
S_{n} & & S_{1} & S_{0}
\end{array}\right], \quad n \geq 0 .
$$

Then $S \in \mathcal{S}_{\kappa}(\mathcal{U}, \mathcal{Y})$ for some $\kappa \geq 0$, if and only if there exists $n_{0}$ positive integer with the property that

$$
\kappa^{-}\left(I-T_{n} T_{n}^{\sharp}\right)=\kappa, \quad \text { for all } n \geq n_{0} .
$$


Proof. Since $\kappa^{-}[\mathcal{U}]=\kappa^{-}[\mathcal{Y}]<\infty$, we can assume without loss of generality that $\mathcal{U}=\mathcal{Y}$. If $S \in \mathcal{S}_{\kappa}(\mathcal{U})$, then a straigtforward calculation shows that

$$
K_{S}(w, z)=\sum_{m, k=0}^{\infty} C_{m k} z^{m} w^{k}
$$

in a neighbourhood of the origin with $\left[C_{m k}\right]_{m, k=0}^{n}=I-T_{n} T_{n}^{\sharp}$, and hence (3.2) holds.

Conversely, the only problem is to show that under condition (3.2) it follows that $S$ is holomorphic in a neighbourhood of the origin. Indeed, if we knew that $S$ is holomorphic in a neighbourhood of the origin, then $S$ would belong to $\mathcal{S}_{\kappa}(\mathcal{U})$, e.g. by [3], Theorem 5.3.16. Define the operators $H_{0}, H_{1}, H_{2}, \ldots$, by the relations: $H_{0}=I, H_{1}=S_{0}$,

$$
H_{n}=S_{n-1}+H_{1} S_{n-2}+\ldots+H_{n-1} S_{0}, \quad n>1 .
$$

Then, it is well-known (see e.g. 11]) that for all $n \geq 0$ we have

$$
M_{n}=\left[\begin{array}{ccccc}
I & H_{1}^{\sharp} & & & H_{n}^{\sharp} \\
H_{1} & I & H_{1}^{\sharp} & & \\
& H_{1} & I & \ddots & \\
& & \ddots & \ddots & \\
H_{n} & & & & I
\end{array}\right]=B_{n}\left[\begin{array}{cc}
I_{\mathcal{U}} & 0 \\
0 & I-T_{n-1} T_{n-1}^{\sharp}
\end{array}\right] B_{n}^{\sharp},
$$

where $B_{n}$ are certain invertible matrices with operator entries. As a consequence, for $n>n_{0}$,

$$
\kappa^{-}\left(M_{n}\right)=\kappa+\kappa^{-}[\mathcal{U}]<\infty
$$

We now make use of an operator version of the construction in [8], Section 19. We consider the complex vector space $\mathcal{F}_{0}(\mathcal{U})$ of all functions $h: \mathbb{Z} \rightarrow \mathcal{U}$ with finite support and, associated to the hermitian kernel

$$
H(i, j)= \begin{cases}H_{i-j} & i \geq j, \\ H_{i-j}^{\sharp} & j>i,\end{cases}
$$

we consider the inner product space $\left(\mathcal{F}_{0}(\mathcal{U}),[\cdot, \cdot]_{H}\right)$, where

$$
[f, g]_{H}=\sum_{i, j \in \mathbb{Z}}[H(i, j) f(j), g(i)], \quad f, g \in \mathcal{F}_{0}(\mathcal{U}) .
$$

We identify $\mathcal{U}$ with the subspace of $\mathcal{F}_{0}(\mathcal{U})$ consisting of all functions $h: \mathbb{Z} \rightarrow \mathcal{U}$ with the property $h(n)=0$ for all $n \neq 0$. Note that this is an embedding of $\mathcal{U}$ into $\mathcal{F}_{0}(\mathcal{U})$ which is isometric, due to the definition of the inner product $[\cdot, \cdot]_{H}$ on $\mathcal{F}_{0}(\mathcal{U})$.

On the space $\mathcal{F}_{0}(\mathcal{U})$ define two operators $S_{+}$and $S_{-}$by: $\left(S_{+}\right) h(n)=h(n-1)$, $\left(S_{-}\right) h(n)=h(n+1)$ for all $h \in \mathcal{F}_{0}(\mathcal{U})$. Clearly, $S_{+} S_{-}=S_{-} S_{+}=$the identity operator on $\mathcal{F}_{0}(\mathcal{U})$, and $\left[S_{+} f, S_{+} g\right]_{H}=[f, g]_{H}, f, g \in \mathcal{F}_{0}(\mathcal{U})$. From (3.6) and Theorem 1.4 in [8], we deduce that $\mathcal{F}_{0}(\mathcal{U})$ can be completed to a Pontryagin space $\mathcal{K}$. More precisely, let $\mathcal{F}_{0}(\mathcal{U})^{0}=\left\{h \in \mathcal{F}_{0}(\mathcal{U}) \mid[h, k]_{H}=0, k \in \mathcal{F}_{0}(\mathcal{U})\right\}$ be the isotropic subspace of $\mathcal{F}_{0}(\mathcal{U})$. There exists a naturally defined inner product, denoted 
also by $[\cdot, \cdot]_{H}$, on the factor space $\mathcal{F}_{0}(\mathcal{U}) / \mathcal{F}_{0}(\mathcal{U})^{0}$ and this inner product space is completed to a Pontryagin space $\mathcal{K}$. Let us note that $\mathcal{U}$ (considered as a subspace of $\left.\mathcal{F}_{0}(\mathcal{U})\right)$ is a direct summand of $\mathcal{F}_{0}(\mathcal{U})^{0}$, since it is nondegenerate. This implies that $\mathcal{U}$ is embedded isometrically into the factor space $\mathcal{F}_{0}(\mathcal{U}) / \mathcal{F}_{0}(\mathcal{U})^{0}$ and hence $\mathcal{U}$ is naturally identified with a (Pontryagin) subspace of $\mathcal{K}$. Clearly, $\kappa^{-}[\mathcal{K}]=\kappa+\kappa^{-}[\mathcal{U}]$. Since the domain and the range of the isometry $S_{+}$are dense in $\mathcal{K}$, it follows that $S_{+}$can be extended by continuity to a unitary operator $U$ on $\mathcal{K}$ and we can easily check that $H_{n}=P_{\mathcal{U}} U^{n} \mid \mathcal{U}$ for all integers $n$, and that $\mathcal{K}=\bigvee_{n \in \mathbb{Z}} U^{n} \mathcal{U}$. With respect to the decomposition $\mathcal{K}=(\mathcal{K} \ominus \mathcal{U}) \oplus \mathcal{U}$, the operator $U$ has the matrix representation $\left[\begin{array}{cc}X & Y \\ T & Q\end{array}\right]$. Then, the transfer map of the unitary system

$$
\left\{\begin{array}{l}
x_{n+1}=X x_{n}+Y u_{n}, \\
y_{n}=T x_{n}+Q u_{n},
\end{array} \quad n \in \mathbb{Z},\right.
$$

is shown to have as its Taylor coefficients about the origin precisely the coefficients of the formal power series of $S$ (the argument is similar to the one in the last part of the proof of Theorem 3.1 and can be omitted; details can be found in [7]). In particular, the formal power series $S$ converges in a neighbourhood of the origin.

This result shows that the Schur classes considered in [2] behave well with respect to coefficient characterizations, in case the coefficient spaces $\mathcal{U}$ and $\mathcal{Y}$ are Pontryagin spaces with the same finite negative index, but the question whether this result holds for the case when $\mathcal{U}$ and $\mathcal{Y}$ are genuine Kreı̆n spaces remains open.

\section{Carathéodory type interpolation problems}

An application of Theorem 3.1 refers to a Carathéodory type problem for meromorphic functions. In this section we let $\mathcal{U}=\mathbb{C}^{m}$ and $\mathcal{Y}=\mathbb{C}^{n}$. The following generalization of the Carathéodory problem was formulated in [10].

Generalized Carathéodory Problem. Let $\left\{S_{l}\right\}_{l=0}^{k}$ be a set of $n \times m$ matrices. It is required to find conditions in order for there to exist functions $G \in \mathcal{S}_{\kappa}\left(\mathbb{C}^{m}, \mathbb{C}^{n}\right)$ with the property that the first $k+1$ Taylor coefficients of $G$ about the origin are, in this order, $S_{0}, S_{1}, \ldots, S_{k}$.

A weaker version of this problem can be formulated, in which the solution is not required to be holomorphic at the origin. More precisely, we can formulate the following problem.

Generalized Carathéodory Problem (Version II). Let $\left\{S_{l}\right\}_{l=0}^{k}$ be a set of $n \times$ $m$ matrices. Given a nonnegative integer $\kappa$, it is required to find conditions in order for there to exist functions $G$ admitting a factorization $G=F B^{-1}$, where $F \in \mathcal{S}_{0}\left(\mathbb{C}^{m}, \mathbb{C}^{n}\right)$ and $B$ is a Blaschke-Potapov product of order $\kappa$, such that the first $k+1$ Taylor coefficients of $F$ about the origin coincide respectively with the first $k+1$ Taylor coefficients about the origin of the function $\left(S_{0}+S_{1} z+\ldots+S_{k} z^{k}\right) B(z)$.

The latter version was completely solved; see [1], [4]. Associated to the data $\left\{S_{0}, S_{1}, \ldots, S_{k}\right\}$, there is a lower triangular Toeplitz block-matrix $T_{k}$ as in (3.1). Using the generalization of the commutant lifting theorem of Sarason-Sz.-Nagy-Foiaş as in [4], it can be shown that the second version of the generalized Carathéodory 
problem is solvable if and only if $\kappa^{-}\left(I-T_{k} T_{k}^{*}\right) \leq \kappa$. Information about the solvability of the first version of the generalized Carathéodory problem can be obtained in terms of the invertibility of $B(0)$; however, a result directly in terms of the data $\left\{S_{0}, S_{1}, \ldots, S_{k}\right\}$ would be of interest. We can obtain such a result in the following way.

First we formulate a direct consequence of Theorem 3.1 .

Theorem 4.1. Given the $n \times m$ matrices $S_{0}, S_{1}, \ldots, S_{k}$ and a nonnegative integer $\kappa$, the following assertions are equivalent:

(i) There exists a function $G \in \mathcal{S}_{\kappa}\left(\mathbb{C}^{m}, \mathbb{C}^{n}\right)$ with the property that the first $k+1$ Taylor coefficients of $G$ about the origin are respectively $S_{0}, S_{1}, \ldots, S_{k}$.

(ii) There exists an infinite sequence $\left\{\tilde{S}_{l}\right\}_{l \geq 0}$ of $n \times m$ matrices such that $\tilde{S}_{l}=S_{l}$ for $l=0,1, \ldots, k$ and there exists $n_{0} \geq 0$ with the property that the matrices $I-\tilde{T}_{n}^{*} \tilde{T}_{n}$ have exactly $\kappa$ negative eigenvalues, counted with their multiplicities, for all $n \geq n_{0}$, where $\tilde{T}_{n}$ are the lower triangular block-Toeplitz matrices associated to the sequence $\left\{\tilde{S}_{l}\right\}_{l \geq 0}$ as in (3.1).

In other words, Theorem 4.1 reduces the problem of finding a solution of the first version of the generalized Carathéodory problem to a problem of infinite completion of a lower triangular block Toeplitz matrix with the additional constraint on the number of negative eigenvalues. This problem was considered in [6] and a solution was found in the case $\kappa=\kappa^{-}\left(I-T_{k}^{*} T_{k}\right)$.

We can now obtain our main result on the generalized Carathéodory problem. For $T \in \mathcal{L}\left(\mathbb{C}^{m}, \mathbb{C}^{n}\right)$, we define the defect operator of $T$ by $D_{T}=\left|I-T^{*} T\right|^{1 / 2}$. Also, $P_{\operatorname{ker} T}$ denotes the orthogonal projection onto the kernel of the operator $T$.

Theorem 4.2. Let $\left\{S_{l}\right\}_{l=0}^{k}$ be a given set of $n \times m$ matrices and $\kappa=\kappa^{-}\left(I-T_{k} T_{k}^{*}\right)$. Then there exists a function $G \in \mathcal{S}_{\kappa}\left(\mathbb{C}^{m}, \mathbb{C}^{n}\right)$ with the property that the first $k+1$ Taylor coefficients of $G$ about the origin are respectively $S_{0}, S_{1}, \ldots, S_{k}$, if and only if

$$
\operatorname{Range}\left(T_{k-1} P_{\text {ker } D_{T_{k-1}}}\left[\begin{array}{c}
S_{1} \\
S_{2} \\
\vdots \\
S_{k}
\end{array}\right]\right)=\operatorname{Range}\left(P_{\text {ker } D_{T_{k-1}^{*}}}\left[\begin{array}{c}
S_{k}^{*} \\
S_{k-1}^{*} \\
\vdots \\
S_{1}^{*}
\end{array}\right]\right) \text {. }
$$

Proof. Theorem 6.4 in [6], Part II, can be applied to the present situation in the form of Corollary 6.8 in [6], Part II, and obtain that (4.1) is a necessary and sufficient condition in order for there to exist a sequence $\left\{\tilde{S}_{p}\right\}_{p \geq 0}$, an infinite continuation of $\left\{S_{l}\right\}_{l=0}^{k}$, with the property that for all $n \geq k$, we have $\kappa^{-}\left(\left\{\tilde{S}_{p}\right\}_{p \geq 0}\right)=\kappa^{-}\left(I-T_{k} T_{k}^{*}\right)$. Then, an application of Theorem 4.1 concludes our proof.

\section{ACKNOWLEDGEMENTS}

We thank J. Rovnyak for several remarks on a preliminary version of the manuscript of this paper, leading to an improvement of the presentation; he informed us that a result related to Theorem 2.1 was considered in an unpublished manuscript by A. Dijksma, M. Dritschel and J. Rovnyak. We also thank to one of the referees for useful remarks concerning the presentation of the last section. 


\section{REFERENCES}

[1] V.M. Adamjan, D.Z. Arov, M.G. KreǏn: Analytic properties of the Schmidt pairs of a Hankel operator and the generalized Schur-Takagi problem, Mat. Sb. (N.S), 86(128)(1971), 34-75; English transl.: Math USSR-Sb., 15(1971), 31-73. MR 45:7505]

[2] D. Alpay, A. Dijksma, J. Rovnyak, H.S.V. De Snoo: Schur Functions, Operator Colligations, and Reproducing Kernel Pontryagin Spaces, Birkhäuser, Basel-Boston-Berlin, 1996. CMP 97:17

[3] T.Ya. Azizov, I.S. Iokhvidov: Linear Operators in Spaces with an Indefinite Metric, Wiley\&Sons, 1989. MR 90j:47042

[4] J.A. Ball, J.W. Helton: A Beurling-Lax theorem for the Lie group $U(m, n)$ which contains most interpolation theory, J. Operator Theory, 9(1983), 107-142. MR 84m:47046

[5] G. Christner, J. Rovnyak: Julia operators and the Schur algorithm, in Harmonic Analysis and Operator Theory, Contemporary Mathematics, Vol. 189, 1995, pp. 135-160. MR 96h:47022

[6] T. Constantinescu, A. Gheondea: Minimal signature in lifting of operators.I, J. Operator Theory, 22(1989), 345-367; II, J. Functional Analysis, 103(1992), 317-351. MR 93c:47041

[7] T. Constantinescu, A. Gheondea: Kolmogorov decompositions and the realization of time dependent systems, preprint 1997.

[8] I.S. IoHvidov, M.G. KREǏN : Spectral theory of operators in indefinite metric.II, Trud. Mosk. Mat. Obshch., 9(1959), 413-496; English transl., Amer. Math. Soc. Transl.(2), 34(1963), 283373. MR 21:6543

[9] M.G. KreĬN, H. Langer: Über die verallgemeinerten Resolventen und die characteristische Funktion eines isometrischen Operators im Raume $\Pi_{\kappa}$, in Hilbert Space Operators and Operator Algebras (Proc. Internat. Conf. Tihany, 1970), Colloq. Math. Soc. Janos Bolyai, Vol.5, Norht Holland, Amsterdam, 1972. MR 54:11103

[10] M.G. KReǏn, H. LANGeR: Über einige Fortsetzungsprobleme, die eng mit der Theorie hermitescher Operatoren im Raume $\Pi_{\kappa}$ zusammenhängen. I. Einige Funktionenklassen und ihre Darstellungen, Math. Nachr., 77(1977), 187-236; II. Verallgemeinerte Resolventen, uResolventen und ganze Operatoren, J. Functional Analysis, 30(1978), 390-447. MR 57:1173

[11] M.G. KreǏn, A.A. Nudelman: The Markov Moment Problem and Extremal Problems, Amer. Math. Soc. Transl. Math. Monographs, Providence, RI, 1977. MR 56:16284

Department of Mathematics, University of Texas at Dallas, Richardson, Texas 75083-0688

E-mail address: tiberiu@utdallas.edu

Institutul de Matematică Al Academiei Române, CP 1-764, 70700 Bucureşti, România

E-mail address: gheondea@imar.ro 